\title{
Phase and Baseline Calibration for Microwave Interferometric Radiometers Using Beacons
}

\author{
Ahmed Kiyoshi Sugihara El Maghraby ${ }^{\circledR}$, Hyuk Park ${ }^{\circledR}$, Adriano Camps ${ }^{\circledR}$, \\ Angelo Grubišić ${ }^{\circledR}$, Camilla Colombo ${ }^{\circledR}$, and Adrian Tatnall
}

\section{Dedicated to the work of Angelo Grubišić}

\begin{abstract}
The relative phase and amplitude calibration between individual receiving elements are one of the key challenges in interferometric microwave radiometry. For monolithic interferometers, for example the Microwave Imaging Radiometer with Aperture Synthesis (MIRAS) on board the Soil Moisture and Ocean Salinity (SMOS) mission, the calibration of these two parameters is accomplished by correlated noise injection, where a noise signal from a central noise source is distributed to multiple elements in phase, and any measured phase and amplitude differences can be measured and compensated. As new multisatellite interferometers are being proposed, a new strategy capable of calibrating these errors between the free-flying antennas as well as any antenna position errors is required. In this article, a new calibration scheme is proposed which uses a set of microwave beacons in place of the central noise source, placed at known locations within the interferometer's field of view. The visibility function produced by these point-sources can be precalculated, and any errors between the calculated and the measured visibility samples can be attributed to the errors to be determined. Since the phase of the measured visibility is a function of phase and antenna position errors, this technique is capable of calibrating these two parameters simultaneously. Calibration equations for far- and near-field beacons are presented. Using these expressions, five interferometric calibration routines are proposed and examined for geostationary formation flight microwave radiometers. Although this technique is ideal for multisatellite interferometers with variable antenna positions, it is also applicable to monolithic interferometers that can undergo substantial array deformation.
\end{abstract}

Index Terms-Atmospheric sounding, calibration, satellite formation flight, synthetic aperture imaging.

\section{INTRODUCTION}

$\mathbf{M}$ ICROWAVE radiometry is a remote sensing technique to measure the brightness temperature of the Earth

Manuscript received January 21, 2019; revised June 16, 2019; accepted September 13, 2019. Date of publication February 5, 2020; date of current version July 22, 2020. This work was supported by the U.K. Engineering and Physical Sciences Research Council (EPSRC) under Award 1503202. (Corresponding author: Ahmed Kiyoshi Sugihara El Maghraby.)

Ahmed Kiyoshi Sugihara El Maghraby and Adrian Tatnall are with the Astronautics Research Group, University of Southampton, Southampton SO17 1BJ, U.K. (e-mail: asem1g14@soton.ac.uk; art4@soton.ac.uk).

Hyuk Park and Adriano Camps are with the CommSensLab, Polytechnic University of Catalonia, 08034 Barcelona, Spain (e-mail: park.hyuk@tsc.upc.edu; camps@tsc.upc.edu).

Angelo Grubišić, deceased, was with the Astronautics Research Group, University of Southampton, Southampton SO17 1BJ, U.K.

Camilla Colombo is with the Department of Aerospace Science and Technology, Politecnico di Milano, 20133 Milan, Italy (e-mail: camilla.colombo@polimi.it).

Color versions of one or more of the figures in this article are available online at http://ieeexplore.ieee.org.

Digital Object Identifier 10.1109/TGRS.2019.2949891 as it naturally radiates through thermal and other radiating mechanisms [1]. With the appropriate understanding of these mechanisms, the measured brightness temperature can be used to recover useful meteorological information of the Earth and its atmosphere, including land and sea surface temperatures [2], [3], soil moisture and ocean salinity [4], [5], sea surface wind [6], as well as atmospheric temperature and humidity profiles [7], amongst other observables.

One of the fundamental challenges behind microwave radiometry is Rayleigh's diffraction limit: due to the long wavelengths at microwave bands, as compared to other key remote-sensing bands, for example, infra-red and visible bands, the achievable spatial resolution can only be improved by increasing the size of the collecting aperture. For cases where the required aperture sizes exceed those achievable using real aperture antennas, interferometric radiometry becomes the only practical option to obtain the required spatial resolution. Originally developed for radio astronomy [8] and later adapted for Earth observation via the Soil Moisture and Ocean Salinity (SMOS) mission [5], an interferometer uses a set of tens to hundreds of small antennas to synthesize a single large aperture. Successful aperture synthesis requires the knowledge of the positions of the individual antennas at accuracies much better than the observed wavelength, and the phase errors between these antennas must not exceed a few degrees [9]. Maintaining these conditions is then the main challenge behind interferometers, and this is achieved through regular calibration.

Recently, to further expand the achievable aperture sizes to those unobtainable using single satellites, interferometric radiometry using formation-flying satellites has been proposed [10], bringing into demand a new calibration strategy which is effective when these antennas are in free-flight. In this article, a new calibration routine is proposed, which is capable of finding the antennas' relative positions and their phase errors by using microwave beacons placed in known locations. The performance of these routines is then tested for the geostationary formation-flight radiometer proposed in [10].

\section{Visibility Function AND Its CAlibRATion}

Interferometers do not directly map the brightness temperature of the target, but they measure an intermediary quantity known as the visibility function, where each pair of antennas measures a point-sample of this function. The visibility sampled by the pair of antennas 1 and 2 is related 
to the observed brightness temperature map by [11], [12]

$$
\begin{aligned}
V_{1,2}(u, v, w) \triangleq & \frac{1}{2} \frac{1}{k B}\left\langle b_{1} b_{2}^{*}\right\rangle \\
= & \frac{1}{\sqrt{\Omega_{1} \Omega_{2}}} \iint_{\xi^{2}+\eta^{2}<1} \tilde{T}_{B}(\xi, \eta) \\
& \cdot \tilde{r}\left(-\frac{u \xi+v \eta+w \sqrt{1-\xi^{2}-\eta^{2}}}{f_{0}}\right) \\
& \cdot e^{-2 \pi i\left(u \xi+v \eta+w \sqrt{1-\xi^{2}-\eta^{2}}\right)} d \xi d \eta
\end{aligned}
$$

for the modified brightness temperature map

$$
\tilde{T}_{B}(\xi, \eta)=\frac{\left(T_{B}(\xi, \eta)-T_{r}\right) F_{n 1}(\xi, \eta) F_{n 2}^{*}(\xi, \eta)}{\sqrt{1-\xi^{2}-\eta^{2}}}
$$

where $k$ is Boltzmann's constant, $B$ is the receivers' predetection bandwidth, $b_{1}$ and $b_{2}$ are the complex amplitude of the voltage induced on antennas 1 and 2, respectively, where ()$^{*}$ refers to the complex conjugate operation and \langle\rangle is the expectation operation. $T_{B}(\xi, \eta)$ is the brightness temperature map at the direction cosines $[\xi, \eta]$, and $T_{r}$ is the physical temperature of both receivers. $\Omega_{1,2}$ and $F_{n 1,2}$ are the antenna beam solid angle and normalized voltage pattern, respectively, and $\tilde{r}$ is the fringe-washing function that accounts for spatial decorrelation effects, which depends on the receivers' frequency responses [13].

To recover the brightness temperature map from the measured visibility, (1) must be inverted using the known baseline vectors, with components $u, v$, and $w$. In addition, the set of visibility samples must be calibrated prior to image reconstruction to take into account any receiver relative phase errors. The free-flying multisatellite design of the proposed interferometer means that the baseline vectors rapidly vary, and these are monitored by intersatellite position sensors, which may drift slowly in time. To maintain an accurate knowledge of the baselines, these drifts must be periodically measured.

The calibration of receiver relative phase errors has been previously addressed for SMOS by means of correlated noise injection [9], [14]-[16]. Noise signal from an internal source is distributed to a set of antennas via the noise distribution network. For an ideal network, these signals arrive at the receivers in phase, and the normalized cross correlation product of these signals should be unity. Any phase difference detected in the measured products, i.e., the visibility phase error, can be attributed to the receiver relative phase error.

With a formation-flying array, noise signals cannot be distributed to the receivers in a network of cables. Instead, a correlated signal can be introduced by an external microwave beacon of known position. The ideal visibility phase can be calculated using the beacon's position, the ideal antenna position, and the ideal receiver phase error. In this case, the measured visibility phase error is a function of antenna position errors as well as the receiver phase errors, and the measured visibility phase errors can be used to calibrate both parameters simultaneously. This technique is applied in the field of geodesy and astrometry in very long baseline interferometry (VLBI), where a set of stars with known positions are used to solve the positions of the antenna elements, which map to the precise orientation of the Earth and the movement of the tectonic plates [8], [17].

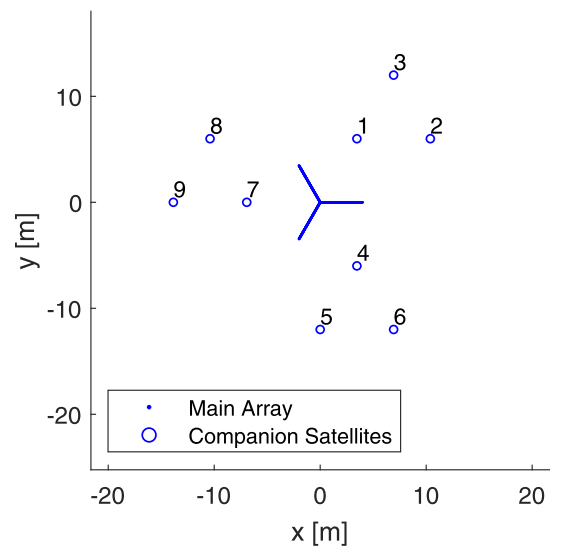

Fig. 1. Proposed antenna distribution for the geostationary formation-flight microwave interferometric radiometer [10].

Now, the formation flight interferometer discussed in this article is described in further detail. Fig. 1 shows the antenna distribution for the proposed interferometer for geostationary atmospheric sounding at $53 \mathrm{GHz}$ [10]. The central Y-shaped array flies in formation with nine companion satellites. The central array contains 236 elements per arm equispaced by $3 \lambda$, leading to a total boom length of $4 \mathrm{~m}$. The nine companion satellites carry one receiver antenna each and are in free flight. Together, the formation synthesizes a circular aperture of $14.4 \mathrm{~m}$ in diameter, leading to $16.7-\mathrm{km}$ spatial resolution from a geostationary orbit. The formation can be maintained via feedback control, where the companion satellites are each actuated by a set of 12 thrusters. The positions of all companion satellites relative to the central satellite can be measured by a set of nine satellite relative position sensors, e.g., the precision laser ranger (PLR) [18], and their orientation can be monitored independently by star trackers at a few-arcsecond accuracy. The PLR measures the satellite relative positions with $40-\mu \mathrm{m}$ precision, which is less than $1 \%$ of the observed wavelength. This sensor, however, may drift slowly in time, and the purpose of this article is to explore possible calibration strategies to periodically measure this drift, as well as the mechanical deformation of the central array, and the drift in the relative phase of all receivers. This article presents a calibration method to achieve this using a set of beacons placed on Earth. Then, the merit of placing some beacons on the formation-flying satellites themselves is explored to improve the accuracy of relative position determination.

The calibration problem is defined as illustrated in Figs. 2 and 3. A set of $N$ antennas are distributed with true position vectors $\left(x_{n}, y_{n}, z_{n}\right)$, and their local oscillator signals are offset by a phase $\phi_{n}$, both with respect to a given reference antenna. The readings from the satellite relative position sensors give the ideal positions of the antennas $\left(x_{\circ n}, y_{\circ n}, z_{\circ n}\right)$. The ideal values for these parameters drift over time due to the instrumental drift of the sensors, and they will be different from the true value. The task of the calibration process is to determine these errors for each receiving element.

\section{CAlibration EQuations}

The calibration technique is based on two calibration equations, where one is applicable for far-field beacons, and 


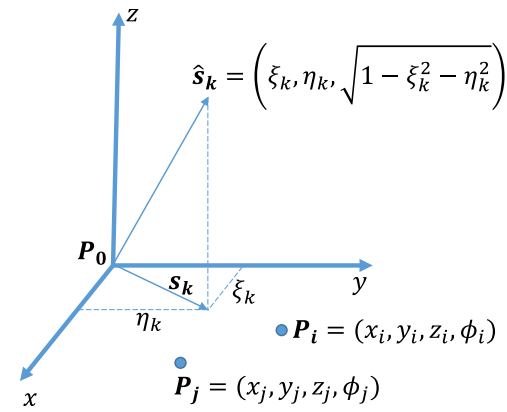

Fig. 2. Point-source and antenna configuration for far-field point sources.

the second for near-field beacons. For both equations, the ideal visibility phase can be calculated for a given beacon and a given baseline. If the antenna position and/or the receiver relative phase contain errors, the measured visibility phase will differ from the ideal values. This error is then used to deduce the unknowns, which are the antenna position error, receiver phase error, and the beacon position error. The two equations are as follows.

\section{A. Far-Field Point Sources}

The position of a far-field point-source can be specified by two direction cosines $(\xi, \eta)$, as shown in Fig. 2. The ideal phase of the visibility measured between antennas $i$ and $j$ for a point-source $k$ is the phase difference anticipated between the two antennas, as follows:

$$
\begin{aligned}
\Phi_{\circ i j k}=- & 2 \pi\left(u_{\circ i j} \xi_{\circ k}+v_{\circ i j} \eta_{\circ k}+w_{\circ i j} \sqrt{1-\xi_{\circ k}^{2}-\eta_{\circ k}^{2}}\right) \\
& +\phi_{\circ i j} \\
=- & \frac{2 \pi}{\lambda}\left(\left(x_{\circ j}-x_{\circ i}\right) \xi_{\circ k}+\left(y_{\circ j}-y_{\circ i}\right) \eta_{\circ k}\right. \\
& \left.\quad\left(z_{\circ j}-z_{\circ i}\right) \sqrt{1-\xi_{\circ k}^{2}-\eta_{\circ k}^{2}}\right)+\left(\phi_{\circ j}-\phi_{\circ i}\right)
\end{aligned}
$$

where the subscript $\circ$ denotes the ideal value for the position and phase of the antennas and the location of the beacon. Due to drift, the true values will be different from the ideal ones by some error $\Delta x, \Delta y, \Delta z, \Delta \phi, \Delta \xi$, and $\Delta \eta$ such that $x_{i}=x_{\circ i}+\Delta x_{i}$ and so on. These differences will offset the measured visibility phase from the ideal value (3), as follows:

$$
\begin{aligned}
\Delta \Phi_{i j k}=-\frac{2 \pi}{\lambda} & \left(\left(\Delta x_{j}-\Delta x_{i}\right) \xi_{\circ k}+\left(\Delta y_{j}-\Delta y_{i}\right) \eta_{\circ k}\right. \\
& \left.+\left(\Delta z_{j}-\Delta z_{i}\right) \sqrt{1-\xi_{\circ k}^{2}-\eta_{\circ k}^{2}}\right)+\left(\Delta \phi_{j}-\Delta \phi_{i}\right) \\
-\frac{2 \pi}{\lambda} & {\left[\Delta \xi_{k}\left(\left(x_{\circ j}-x_{\circ i}\right)-\frac{\xi_{\circ k}\left(z_{\circ j}-z_{\circ i}\right)}{\sqrt{1-\xi_{\circ k}^{2}-\eta_{\circ k}^{2}}}\right)\right.} \\
& \left.+\Delta \eta_{k}\left(\left(y_{\circ j}-y_{\circ i}\right)-\frac{\eta_{\circ k}\left(z_{\circ j}-z_{\circ i}\right)}{\sqrt{1-\xi_{\circ k}^{2}-\eta_{\circ k}^{2}}}\right)\right] .
\end{aligned}
$$

This is the general calibration equation for a far-field beacon and a pair of receiver antennas. A set of $N$ antennas and $M$ beacons will generate a set of $M N(N-1) / 2$ visibility samples, from which a coefficient matrix can be constructed for the system of calibration equations. In practice, this equation will be simplified with some a priori knowledge, provided either by measurement or by definition as reference.

For argument, let $N=N_{1}+N_{2}+1$ be the total number of antennas, where $N_{2}$ is the number of antennas only the phase of which requires calibration, while their positions are known with sufficient accuracy, and $N_{1}$ is the number of antennas both phase and position of which require calibration. The remaining antenna is the reference antenna. Then, let $M=M_{1}+M_{2}$ be the total number of beacons, where $M_{2}$ is the number of beacons with known positions, and $M_{1}$ is the number of beacons with uncertain positions.

With the above, a truncated direction vector $\mathbf{s}=(\xi, \eta)$ can be defined, such that

$$
\Delta \mathbf{s}_{k}=\left[\Delta \xi_{k}, \Delta \eta_{k}\right], \quad \Delta \mathbf{P}_{i}=\left[\Delta x_{i}, \Delta y_{i}, \Delta z_{i}, \Delta \phi_{i}\right]
$$

and the coefficient matrix $\mathbf{M}$ can be constructed as follows:

$$
\Delta \Phi=\mathbf{M}\left[\begin{array}{l}
\Delta \mathbf{P} \\
\Delta \phi \\
\Delta \mathbf{S}
\end{array}\right]
$$

where $\Delta \Phi$ is a vector of length $M N(N-1) / 2$, containing the phase errors for all visibility samples measured, $\Delta \mathbf{P}$ is a vector of length $4 N_{1}$, containing the unknown positions and phases for $N_{1}$ antennas, $\Delta \phi$ is a vector of length $N_{2}$, containing the unknown phases for $N_{2}$ antennas, and $\Delta \mathbf{s}$ is a vector of length $2 M_{1}$, containing the unknown positions of $M_{1}$ beacons. The size of the coefficient matrix $\mathbf{M}$, then, is $M N(N-1) / 2$ by $4 N_{1}+N_{2}+2 M_{1}$.

If the necessary conditions are met, the coefficient matrix $\mathbf{M}$ has a full rank and can be inverted to solve the vector of unknowns. As will be further discussed in Section IV-A, the far-field beacons may be placed in the Earth's atmosphere, at a sufficient altitude to avoid atmospheric attenuation. This means that Global Navigation Satellite System (GNSS) can be used to locate these beacons at accuracies much higher than the interferometer's spatial resolution, thus $\Delta \mathbf{s}$ will be negligible for all beacons. In this condition, it is found that a minimum of four beacons will give the coefficient matrix $\mathbf{M}$ a full rank.

\section{B. Near-Field Point Sources}

If the distance to a beacon is comparable to the baseline length, then its 3-D position must be taken into account. Since the receiver elements are in the far-field of the beacon antenna, their wavefronts within the receiver array are considered spherical. Fig. 3 shows the $k^{\text {th }}$ beacon $\mathbf{I}_{k}$ illuminating two antennas $\mathbf{P}_{i}$ and $\mathbf{P}_{j}$. The ideal phase of the visibility measured between these antennas is

$$
\Phi_{\circ i j k}=\frac{2 \pi}{\lambda}\left(\left|\mathbf{r}_{\circ j k}\right|-\left|\mathbf{r}_{\circ i k}\right|\right)+\phi_{\circ i j}
$$

where $\left|\mathbf{r}_{i k}\right|$ is the distance between antenna $i$ and source $k$

$$
\left|\mathbf{r}_{i k}\right|=\sqrt{\left(x_{I k}-x_{i}\right)^{2}+\left(y_{I k}-y_{i}\right)^{2}+\left(z_{I k}-z_{i}\right)^{2}} .
$$




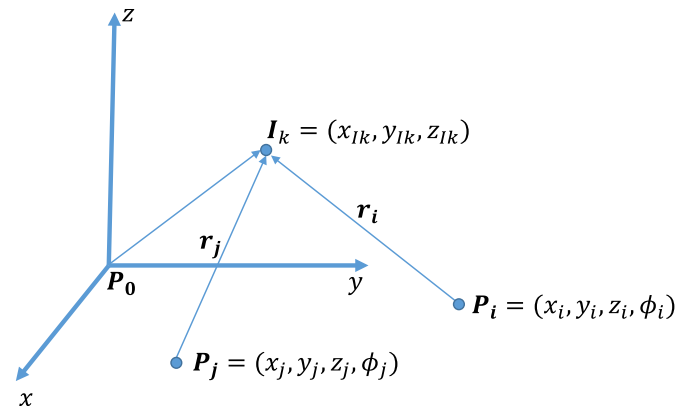

Fig. 3. Point-source and antenna configuration for near-field point sources.

By partially differentiating (7), the measured visibility phase error in terms of the antenna position and phase errors is

$$
\begin{aligned}
\Delta \Phi_{i j k}=-\frac{2 \pi}{\lambda} & \left(\frac{x_{\circ I k}-x_{\circ j}}{\left|r_{\circ j k}\right|} \Delta x_{j}-\frac{x_{\circ I k}-x_{\circ i}}{\left|r_{\circ i k}\right|} \Delta x_{i}\right. \\
& +\frac{y_{\circ I k}-y_{\circ} j}{\left|r_{\circ j k}\right|} \Delta y_{j}-\frac{y_{\circ I k}-y_{\circ i}}{\left|r_{\circ i k}\right|} \Delta y_{i} \\
& \left.+\frac{z_{\circ I k}-z_{\circ j}}{\left|r_{\circ}\right|} \Delta z_{j}-\frac{z_{\circ I k}-z_{\circ i}}{\left|r_{\circ i k}\right|} \Delta z_{i}\right) \\
+\frac{2 \pi}{\lambda} & {\left[\left(\frac{x_{\circ I k}-x_{\circ j}}{\left|r_{\circ j k}\right|}-\frac{x_{\circ I k}-x_{\circ i}}{\left|r_{\circ i k}\right|}\right) \Delta x_{I k}\right.} \\
& +\left(\frac{y_{\circ I k}-y_{\circ j}}{\left|r_{\circ j k}\right|}-\frac{y_{\circ I k}-y_{\circ i}}{\left|r_{\circ i k}\right|}\right) \Delta y_{I k} \\
& \left.+\left(\frac{z_{\circ I k}-z_{\circ j}}{\left|r_{\circ j k}\right|}-\frac{z_{\circ I k}-z_{\circ i}}{\left|r_{\circ i k}\right|}\right) \Delta z_{I k}\right] \\
+ & \left(\Delta \phi_{j}-\Delta \phi_{i}\right) .
\end{aligned}
$$

Now, this calibration equation can be used to construct a coefficient matrix in the same manner as shown in (6). The key difference is that the vector $\Delta \mathbf{s}$ is now $3 M_{1}$ elements long, as the beacon position is now given by three quantities.

As further discussed in Section IV-B, the near-field beacons may be placed on the free-flying satellites. This means that $\Delta \mathbf{s}$ for all beacons are uncertain, and there is no number of beacons that will fully constrain the coefficient matrix $\mathbf{M}$ using near-field beacons alone. This technique requires a minimum of two additional far-field beacons to give $\mathbf{M}$ a full rank.

\section{Virtual Point SOURCE}

The calibration method described above requires a set of point sources as the only source of radiation in the interferometer's field of view, where one beacon illuminates the array at any given time. This condition cannot be attained in practice, since the interferometer will always be illuminated by the Earth and other celestial bodies. The visibility of a point source, however, can be generated by first illuminating the interferometer with a beacon and the surrounding environment, followed by a second illumination of equal integration time with the beacon switched OFF. The difference between the two visibility samples is then

$$
V_{c a l}=\left(V_{\mathrm{env} 1}+V_{\mathrm{bcn}}\right)+n_{1}-V_{\mathrm{env} 2}+n_{2}
$$

where $V_{\text {cal }}$ is the measured visibility for calibration, and $V_{\text {env1 }}$ and $V_{\text {env2 }}$ are the contributions to the measured visibility by the environmental illumination for the first and the second exposures, respectively. $V_{\mathrm{bcn}}$ is the contribution by the beacon, illuminating the antennas during the first illumination. The power of this contribution is found as

$$
\left|V_{\mathrm{bcn}, i j}\right|=\frac{\sqrt{P_{\mathrm{bcn}, i} P_{\mathrm{bcn}, j}}}{k B} \cdot\left|\tilde{r}\left(B \tau_{\Delta, i j}\right)\right|
$$

where $P_{\mathrm{bcn}, i}$ and $P_{\mathrm{bcn}, j}$ are the power received by antennas $i$ and $j$ from the beacon, with signals reaching the two antennas with $\tau_{\Delta, i j}$ delay. $\tilde{r}()$ is the fringe-washing function.

$n_{1}$ and $n_{2}$ are the complex-valued zero-mean additive noise remaining in the visibility due to finite integration time. If the integration time is sufficiently short, such that the observed brightness temperature map does not change during the two observations, then $V_{\text {env1 }}=V_{\text {env2 }}$ can be ensured, and the remaining noise in the measured visibility will be $n_{1}+n_{2}$. Since $n_{1}$ and $n_{2}$ are independent stochastic processes, the variance of the sum $n_{1}+n_{2}$ is the sum of the individual variances. Following [11] and [19], the real and imaginary components of the visibility noise variance for the first illumination, with double-sideband receivers are:

$$
\begin{array}{r}
\sigma_{n_{1, \mathrm{re}}}^{2}=\frac{1}{2 B \tau_{\mathrm{eff}}}[ \\
\quad\left(T_{A i}+T_{R i}\right)\left(T_{A j}+T_{R j}\right) \\
\left.+V_{i j, \mathrm{bcn}, \mathrm{re}}^{2}-V_{i j, \mathrm{bcn}, \mathrm{im}}^{2}\right] \\
\sigma_{n_{1, \mathrm{~m}}}^{2}=\frac{1}{2 B \tau_{\mathrm{eff}}}[ \\
\left(T_{A i}+T_{R i}\right)\left(T_{A j}+T_{R j}\right) \\
\left.+V_{i j, \mathrm{bcn}, \mathrm{im}}^{2}-V_{i j, \mathrm{bcn}, \mathrm{re}}^{2}\right]
\end{array}
$$

for receiver noise temperatures $T_{R}$ and antenna temperatures $T_{A}$ for antennas $i$ and $j$, where the antenna temperature contains the contribution of the environmental illumination and that of the beacon, given as

$$
T_{A i}=T_{\mathrm{env}, i}+T_{\mathrm{bcn}, i}=T_{\mathrm{env}, i}+\frac{P_{\mathrm{bcn}, i}}{k B}
$$

for the $i^{\text {th }}$ antenna, where $T_{\mathrm{env}, i}$ is the brightness temperature contribution of the Earth and the other celestial bodies, and $P_{\mathrm{bcn}, i}$ is the power received by antenna $i$ from the beacon. $\tau_{\text {eff }}$ is the effective integration time depending on the bit-depth and sampling frequency of the receiver [19], [20]. For 1-bit $2 B$ sampling, $\tau_{\text {eff }}=\tau / 2.46$.

For the second illumination, the interferometer is illuminated by a continuous source that is the Earth. This means that the real and imaginary visibility components $V_{\text {re }}$ and $V_{\mathrm{im}}$ are generally much smaller than the antenna and receiver temperatures $T_{A}$ and $T_{R}$, and the expressions for the visibility noise can thus be simplified as [21]

$$
\sigma_{n_{2}}^{2}=\sigma_{n_{2, \mathrm{re}}}^{2}=\sigma_{n_{2, \mathrm{im}}}^{2}=\frac{\left(T_{A i}+T_{R i}\right)\left(T_{A j}+T_{R j}\right)}{2 B \tau_{\text {eff }}} .
$$

The residual noise in the phase of the measured visibility for near-field beacons can now be expressed, as follows:

$$
\begin{aligned}
\sigma_{\Delta \Phi}=\frac{1}{\left|V_{\mathrm{bcn}}\right|^{2}}\left[\left(\sigma_{n_{1, \mathrm{re}}}^{2}+\sigma_{n_{2}}^{2}\right)\right. & V_{\mathrm{bcn}, \mathrm{im}}^{2} \\
& \left.+\left(\sigma_{n_{1, \mathrm{im}}}^{2}+\sigma_{n_{2}}^{2}\right) V_{\mathrm{bcn}, \mathrm{re}}^{2}\right]^{\frac{1}{2}}
\end{aligned}
$$

For far-field beacons, (12) becomes

$$
\left|V_{\mathrm{bcn}}\right| \ll T_{A}+T_{R}, \quad \sigma_{n_{1, \mathrm{re}}}^{2}=\sigma_{n_{1, \mathrm{im}}}^{2}=\sigma_{n_{2}}^{2}=\sigma_{n}^{2}
$$


and (15) can be simplified as

$$
\sigma_{\Delta \Phi}=\frac{\sqrt{2 \sigma_{n}^{2}}}{\left|V_{\mathrm{bcn}}\right|} .
$$

For both (15) and (17), $\sigma_{\Delta \Phi}$ is inversely proportional to the square-root of the cumulative integration time. Long integration time can be achieved while maintaining the condition $V_{\text {env1 }}=V_{\text {env2 }}$ by taking pairs of short integrations repeatedly until the required noise level is reached.

Now, the implementation of these beacons is discussed.

\section{A. Beacons on Board High Altitude Balloons}

At sounding frequencies, the beacons must be placed at high altitudes to avoid atmospheric attenuation. Precision pointing onboard high-altitude balloons has several precedents in millimeter, sub-millimeter, and ultraviolet band astronomy, where telescopes were flown on board balloons with apertures of up to $2 \mathrm{~m}$, and have been stabilized at $1 \sigma$ of a few tens of arcseconds [22]-[24].

With these high altitude platforms, the atmospheric attenuation loss is approximately $10 \%$ at $10-\mathrm{km}$ altitude, at the $53-\mathrm{GHz}$ proposed sounding frequency [25].

Note that while the transmitter antenna gain is maintained at its maximum value by allowing the transmitter antenna to track the constellation, the receiver antenna gain on board the interferometer depends on the position of the transmitter, since the receiving antennas will always be pointing toward the Earth's center. With the proposed antenna [10], [26], this gain ranges from $17.8 \mathrm{dBi}$ at boresight to $16.4 \mathrm{dBi}$ at horizon.

\section{B. Beacons Within the Interferometer Constellation}

The alternative solution to placing the beacons on board high altitude platforms is to place them in the vicinity of the multisatellite interferometer. This approach reduces the transmission range by a factor of $10^{6}$ to $10^{7}$, and reduces the required equivalent isotropically radiated power (EIRP) by the square of this quantity. The main challenge in implementing this scheme is twofold, as follows.

1) Both transmitting and receiving antennas will be located at $90^{\circ}$ from boresight, leaving just $-12.2-\mathrm{dBi}$ gain for these antennas. The beam phase pattern must also be accurately known at these extreme angles.

2) As the signals are transmitted and received within the plane of the interferometer, delays of 83 ns can be expected for the longest baselines. With the proposed $400-\mathrm{MHz}$ bandwidth, just $1 \%$ of the received power will remain at the correlator output, due to extreme signal decorrelation, i.e., fringe-washing [27].

Having overcome these challenges, near-field beacons can be implemented in one of the following ways.

1) Selected receiver antennas act as beacons during the calibration phase.

2) A dedicated set of transmitter antennas are attached to the satellites.

3) A dedicated constellation of satellites hosts the beacons.

The first option is particularly attractive from two points of view. First, it requires no additional antennas, and second,

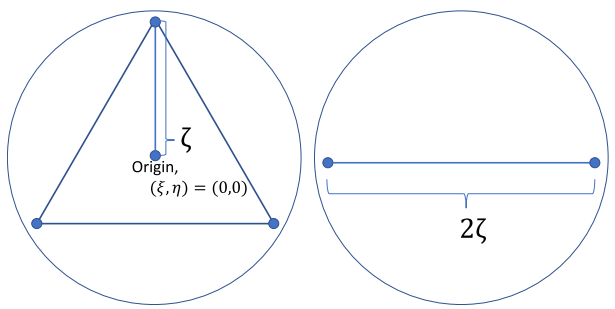

Fig. 4. Two proposed configurations for the airborne beacons. The larger circle shows the Earth's horizon as seen from the geostationary orbit.

because the transmitter antennas are by themselves receiver antennas, this option significantly reduces the number of unknowns, allowing higher levels of overdetermination and improved calibration accuracy.

Given the above, the performance of the first option is explored, where the receiver antennas transmit one at a time, while the remaining receivers calibrate their position and phase. To avoid the extreme fringe-washing effect, the beacons shall transmit a continuous wave at the center frequency of the receivers. Nevertheless, it is useful to maintain the beacons' capability to transmit broadband noise signals to allow the calibration of the fringe-washing function shape, once the antenna positions and phase are calibrated [27], [28].

\section{Example Calibration Routines}

Applying the concepts discussed thus far, five calibration routines are designed, applicable to the multisatellite interferometer proposed in [10]. These routines are categorized into three types. The first, type-A uses a single far-field beacon flown on board a high altitude balloon, to calibrate the phase of all antennas which have known positions. Type-B calibration applies four far-field beacons to calibrate the phase and position of all antennas. This category includes two calibration routines: the truncated routine where some of the antennas have known positions, and the full routine where all antenna positions are calibrated. Type-C applies a combination of near-field and far-field beacons, again including truncated and full calibration routines.

Antenna position errors in the central array occur as a result of mechanical deformation, which will likely happen at a slower rate for much smaller displacements than that for the companion satellites, which are in free flight. Therefore, the full calibration routines will be required at a less frequent rate as the central array deformation exceeds an acceptable threshold. This threshold will be quantified in this section.

Fig. 4 (left) shows the distribution of the four far-field beacons to fully constrain the coefficient matrix $\mathbf{M}$ for type-B calibration, where the larger circle represents the Earth's horizon, and $\zeta$ corresponds to the magnitude of $\mathbf{s}_{k}$ in Fig. 2 for the triangle vertexes. The condition of the coefficient matrix $\mathbf{M}$ can be improved by increasing $\zeta$. Note that a square formation leaves $\mathbf{M}$ underconstrained by one degree of freedom. The performance of this configuration is further discussed in Section V-B. Fig. 4 (right) shows the two-beacon distribution for type- $\mathrm{C}$ calibration, where the introduction of near-field beacons reduces the number of required far-field beacons to two, as further discussed in Section V-C. 
TABLE I

EXAmple Configuration For Receiver Phase CALIBRATION USING ONE FAR-FIELD BEACON

\begin{tabular}{lll}
\hline Parameter & Symbol & Value \\
\hline Transmitter Antenna Diameter & $D_{T}$ & $0.6 \mathrm{~m}$ \\
Transmitter Antenna Gain & $G_{T}$ & $50.4 \mathrm{dBi}$ \\
Transmitter Antenna Radiated Power & $P_{T}$ & $12 \mathrm{~W}$ \\
EIRP & - & $1.3 \times 10^{6} \mathrm{~W}$ \\
Cumulative Integration Time per Beacon & $\tau_{\text {far }}$ & $1 \mathrm{~s}$ \\
Total Calibration Time & $\tau_{\text {tot }}$ & $2 \mathrm{~s}$ \\
Visibility Phase Noise & $\sigma_{\Delta \Phi}$ & $18 \mathrm{mrad}$ \\
Total Calibration Precision & $\sigma_{\Delta \phi}$ & $0.93 \mathrm{mrad}$ \\
\hline
\end{tabular}

The performance of the five calibration routines for the three calibration types is now explored.

\section{A. Phase-Only Calibration}

If the position of all antennas is exactly known, then the set of unknowns is the phase of $N-1$ antennas, excluding the reference antenna. The relationship between the measured visibility phase error $\Delta \Phi$ and the phase error of the individual antennas $\Delta \phi$ is outlined in (4), where $\Delta x, \Delta y, \Delta z, \Delta \xi$, and $\Delta \eta$ are zero. It is found that a single far-field beacon fully constrains the coefficient matrix $\mathbf{M}$.

Method Robustness: With a fully constrained M, residual noise in the recovered $\Delta \phi$ is propagated from the noise in the measured visibility, $\Delta \Phi$. The robustness of the calibration method is examined by quantifying the degree at which this noise propagates into $\Delta \phi$. For a given vector of visibility phase noise $\sigma_{\Delta \Phi}$, with $\mathbf{M}^{-1}$ the pseudoinverse of $\mathbf{M}$, the vector of the recovered phase noise $\sigma_{\Delta \phi}$ is

$$
\sigma_{\Delta \phi}^{2}=\left(\mathbf{M}^{-1}\right)^{\circ 2} \sigma_{\Delta \Phi}^{2}
$$

where ()$^{\circ 2}$ is the element-wise square operation of a matrix.

Given that $\sigma_{\Delta \Phi}$ is dominated by independent thermal noise, the noise variance of the recovered unknowns is proportional to the visibility phase noise variance and inversely proportional to the total number of antennas.

With a Y-shaped array of 236 elements per arm, the remaining $\sigma_{\Delta \phi}$ is constant across all antennas at $53 \mathrm{mrad}$ per $\sigma_{\Delta \Phi}$. To achieve 1-mrad calibration precision, for example, $\sigma_{\Delta \Phi}=$ $18 \mathrm{mrad}$ would be needed, which can be achieved by a configuration summarized in Table I.

\section{B. Receiver Phase and Baseline Calibration Using Airborne Beacons}

To calibrate the antenna positions and phases, the coefficient matrix $\mathbf{M}$ is constructed using (4) for $N$ antennas with $4(N-1)$ unknowns and $M N(N-1) / 2$ equations, where $M=M_{2}$ is the number of far-field beacons with known positions. Minimum of $M=4$ gives $\mathbf{M}$ a full rank.

In the same manner as previously described for phase calibration, the noise variance in the recovered unknowns is proportional to the noise variance in the measured $\Delta \Phi$ and inversely proportional to the total number of antennas. The degree to which the error propagates in this method, however, is also sensitive to the distribution of the beacons. Furthermore, the recovered $\Delta z$ and $\Delta \phi$ are strongly coupled, as also demonstrated in [29]. To account for this coupling, expression (18) is
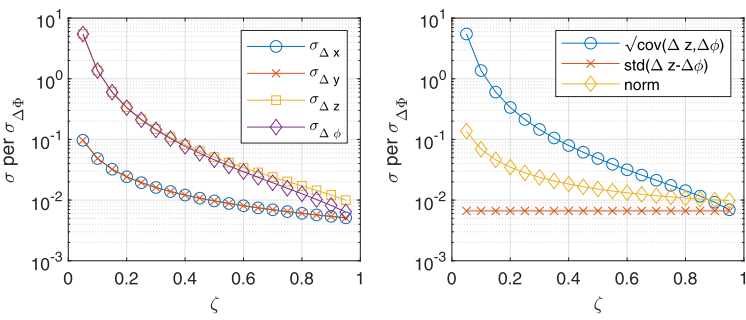

Fig. 5. Achieved calibration precision per measured visibility phase uncertainty for the truncated receiver position and phase calibration using four airborne beacons. The "norm" line in (right) shows the norm of $\sigma_{\Delta x}, \sigma_{\Delta y}$, and $\operatorname{std}(\Delta z-\Delta \phi)$.

generalized using covariance matrices. If the covariance matrix of the measured visibility phase error is $\operatorname{cov}(\Delta \Phi)$, then the covariance matrix of the recovered unknowns $\operatorname{cov}(\Delta \mathbf{P})$ can be found as follows:

$$
\operatorname{cov}(\Delta \mathbf{P})=\mathbf{M}^{-1} \operatorname{cov}(\Delta \Phi)\left(\mathbf{M}^{-1}\right)^{\mathrm{T}}
$$

where $\operatorname{cov}(\Delta \Phi)$ is a diagonal covariance matrix, since $\sigma_{\Delta \Phi}$ are caused by independent thermal processes. Equation (19) is applied to find the achievable calibration accuracy with the beacon distribution shown in Fig. 4 (left) as a function of $\zeta$.

The performance of this calibration type is evaluated for two calibration routines.

1) In the truncated calibration, the antenna positions within the central array are known with sufficient accuracy, and the positions of the formation-flying companion antennas are calibrated, together with the phase of all antennas.

2) In the full calibration, the position and phase of all antennas are calibrated.

The method robustness of these two scenarios is as follows.

1) Truncated Calibration Using Far-Field Beacons: Using four airborne beacons and known positions of central antennas, the positions of companion antennas and the phase of all antennas are calibrated. Note that the available prior knowledge does not reduce the required number of beacons to constrain the coefficient matrix $\mathbf{M}$, since the wavefronts for all beacons are planar. This is in contrast to the case where spherical wavefronts are available for near-field beacons, as later shown in Section V-C2, where the required number of airborne beacons can be reduced to one.

Fig. 5 shows the standard deviation of the noise remaining in the measured unknowns $\sigma_{\Delta x}, \sigma_{\Delta y}, \sigma_{\Delta z}$, and $\sigma_{\Delta \phi}$, together with the square-root of the covariance of $\Delta z$ with $\Delta \phi$, all normalized to $\sigma_{\Delta \Phi}$. The remaining covariances are negligible. The phase $\sigma_{\Delta \phi}$ here is given in cycles, not radians, to better show its coupling with $\sigma_{\Delta z}$. Note that the calibration equation (4) is not dependent on the antenna positions $x, y$, and $z$, for $\Delta \xi=\Delta \eta=0$. This means that the precision shown in Fig. 5 applies to all formation-flying companion antennas.

For the proposed method, it is only possible to position the airborne beacons within $\zeta<0.15$, which is the radius of the Earth as seen from the geostationary orbit. The remaining points are shown to illustrate the merit of placing the beacons on board the formation-flying satellites. For $\zeta<0.15$, all beacons occupy a relatively narrow field of view, and 
TABLE II

Example Configuration for TRuncated Receiver Position and Phase Calibration Using Four Far-Field Beacons

\begin{tabular}{lll}
\hline Parameter & Symbol & Value \\
\hline Transmitter Antenna Effective Diameter & $D_{T}$ & $2 \mathrm{~m}$ \\
Transmitter Antenna Gain & $G_{T}$ & $60.1 \mathrm{dBi}$ \\
Transmitter Antenna Radiated Power & $P_{T}$ & $12 \mathrm{~W}$ \\
EIRP & - & $14.8 \mathrm{MW}$ \\
Cumulative Integration Time per Beacon & $\tau_{\text {far }}$ & $0.5 \mathrm{~s}$ \\
Total Calibration Time & $\tau_{\text {tot }}$ & $4 \mathrm{~s}$ \\
Visibility Phase Noise & $\sigma_{\Delta \Phi}$ & $2.2 \mathrm{mrad}$ \\
& $\sigma_{\Delta x}$ & $71 \mu \lambda$ \\
Total Calibration Precision & $\sigma_{\Delta y}$ & $71 \mu \lambda$ \\
& $\sigma_{\Delta z}$ & $1.3 \mathrm{~m} \lambda$ \\
& $\sigma_{\Delta \phi}$ & $8.3 \mathrm{mrad}$ \\
\hline
\end{tabular}

the coefficient matrix $\mathbf{M}$ is poorly conditioned, leaving $\sigma_{\Delta \phi}$ strongly coupled with $\sigma_{\Delta z}$, at much higher levels than $\sigma_{\Delta x}$ and $\sigma_{\Delta y}$.

Although this means that it is extremely challenging to achieve high calibration precision for $\Delta z$ and $\Delta \phi$ simultaneously, it is relatively easy to calibrate the pair of variables as a coupled variable. The key noise level in this case is the standard deviation of the difference $\operatorname{std}(\Delta z-\Delta \phi)$. This is also plotted in Fig. 5 (right), and the norm of this value together with $\sigma_{\Delta x}$ and $\sigma_{\Delta y}$ is used to find the required $\sigma_{\Delta \Phi}$ in a similar manner previously shown in Section V-A.

To define the required calibration precision, let the relative position sensor on board the constellation be characterized by $1 \sigma$ stability of $40 \mu \mathrm{m}$ [18]. Aiming for approximately an order of magnitude better precision, $\sigma_{\Delta \Phi}$ should be $1 \times 10^{-4} \lambda$ at the proposed 53-GHz center frequency. From Fig. 5 (right), the required $\sigma_{\Delta \Phi}$ to achieve this norm is $2.2 \mathrm{mrad}$ for the proposed array. This can be achieved with the beacon configuration illustrated in Table II.

To verify that this approach restores acceptable radiometric accuracy despite the large residual $\sigma_{\Delta z}$, the formation flight interferometer simulator developed in [10] is applied. With the covariance matrix generated above, a set of random residual errors is generated for each antenna, which is passed to the interferometer simulator to find the resulting degradation in the interferometer radiometric accuracy.

Fig. 6 shows the input image to the simulator, the output image with correct antenna positions and phases (discretization error noise floor), the error map caused by antenna position errors with $\sigma=0.02 \lambda$ and the error map after calibration. The average of 50 Monte Carlo runs are taken for the beacon distribution shown in Fig. 7 with varying $\zeta$ and $\sigma_{\Delta \Phi}$.

Noting that $\Delta T=0.05 \mathrm{~K}$ is the noise floor of the image reconstruction and recovery algorithm, defined as the threshold residual for the iterative CLEAN algorithm [30], Fig. 7 suggests that for all $\zeta$ the beacon configuration presented in Table II shows an excellent calibration performance. The results suggest that the beacon requirements may be relaxed by an order of magnitude with acceptable radiometric accuracy, for example, by reducing the transmitter antenna diameter by a factor of 3. One notable result Fig. 7 reveals is that radiometric accuracy does not improve by separating the airborne beacons by longer distances, as suggested by the norm in Fig. 5 (right). As will be further discussed in Section V-B2, this is due
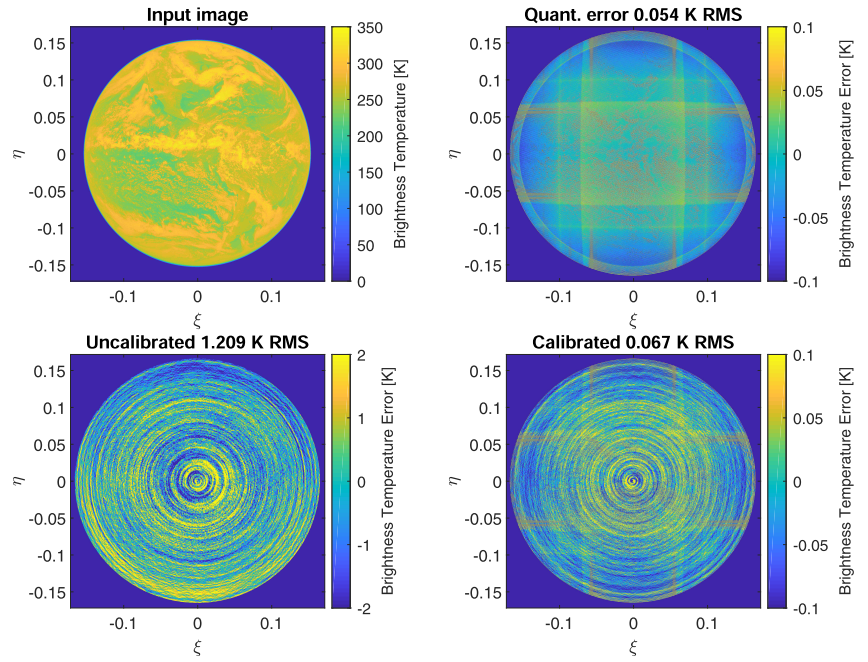

Fig. 6. Example simulation results evaluating the performance of the calibration strategies presented in this article using the multisatellite interferometer simulator [10]. (Bottom left) Error map caused by antenna position errors with $\sigma=0.02 \lambda$. (Bottom right) Error map after calibration.

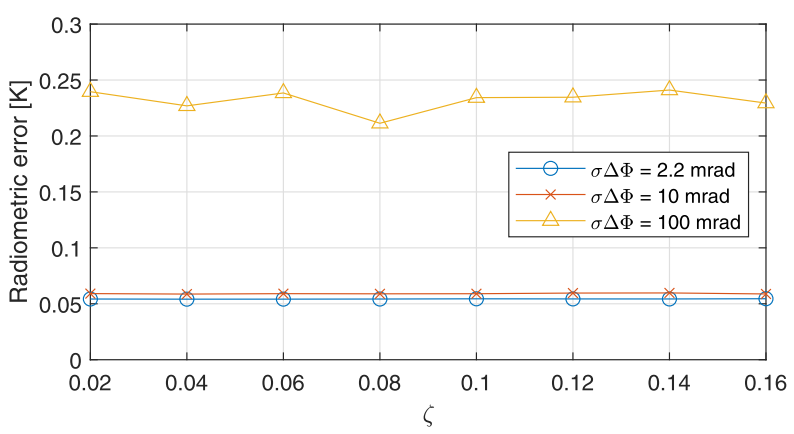

Fig. 7. Simulation results of radiometric accuracy loss caused by residual antenna position and phase errors after calibration, for various levels of visibility phase uncertainty $\sigma_{\Delta \Phi}$ and beacon positions $\zeta$, as shown in Fig. 4, for the truncated calibration routine (average of 50 Monte Carlo runs).
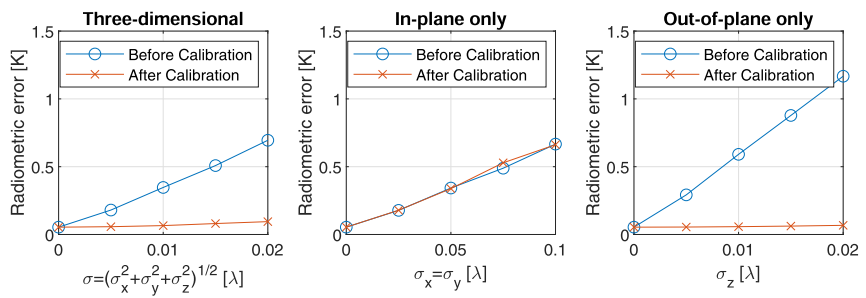

Fig. 8. Simulation results for additional radiometric error caused by central array distortion for the truncated calibration routine, run with $3-\mathrm{D}$, in-planeonly and out-of-plane-only weighted random errors. The truncated calibration routine cannot compensate for in-plane errors.

to the residual out-of-plane errors $\operatorname{std}(\Delta z-\Delta \phi)$ being the dominant cause of radiometric error, which does not improve by increasing $\zeta$.

Finally, recall that this method assumes that the positions of the central elements are accurately known. If the central array is deformed outside our knowledge, the effects are twofold. First, the calibrated positions of the companion antennas will be incorrect. Second, the incorrect positions of these antennas (companion as well as central) introduce strong noise into the recovered images, as illustrated in Fig. 8.

Fig. 8 is produced by adding random errors to the antenna positions of the central array, with in-plane components $\sigma_{x}$ 

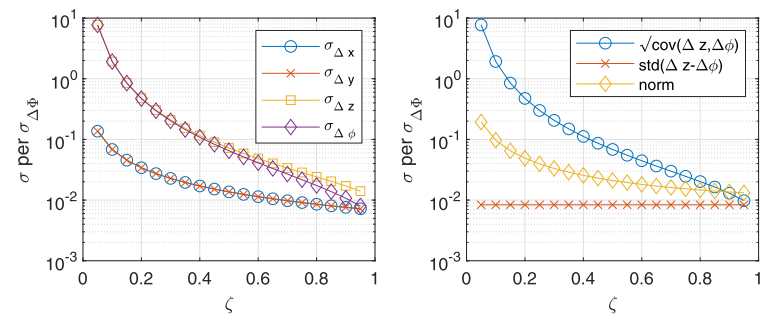

Fig. 9. Achieved calibration precision per measured visibility phase uncertainty for the full receiver position and phase calibration using four airborne beacons.

and $\sigma_{y}$, and out-of-plane component $\sigma_{z}$. These errors are weighted, as suggested in [29], such that the error is zero at the center and increases linearly to $2 \sigma_{x}, 2 \sigma_{y}, 2 \sigma_{z}$, at the boom tips. With these errors, simulation results show similar levels of radiometric accuracy loss as found in [29] prior to calibration, with errors caused mostly by the out-of-plane component $\sigma_{z}$.

Fig. 8 also shows the radiometric accuracy after calibration. To demonstrate the severity of the effects of central antenna position errors, the array is calibrated with infinite integration time being allowed. No errors are given to the companion antennas, leaving the central array deformation as the sole contributor to the loss of radiometric accuracy.

Although the calibration process does not calibrate the antenna positions of the central array nor does it calibrate the positions of the companion antennas correctly, it does manage to compensate for the out-of-plane errors by finding the appropriate phase errors for each antenna. As a result, the calibration process yields substantial radiometric accuracy improvement by reducing the error contribution of $\sigma_{z}$, where residual radiometric error remained at the noise floor of the recovery algorithm for all tested $\sigma_{z}$. The calibration process fails to reduce the contributions of $\sigma_{x}$ and $\sigma_{y}$.

These results show that in addition to the radiometric errors caused by thermal noise $\sigma_{\Delta \Phi}$, shown in Fig. 7, further errors are expected due to central array deformation, where additional error of approximately $0.1 \mathrm{~K}$ for every $\sigma=0.02 \lambda$ [see Fig. 8 (left)] can be expected. To address these results, the full calibration routine is now described, where the positions and phases of all antennas are calibrated.

2) Full Calibration Using Far-Field Beacons: In this routine, the phase and positions of all antennas are calibrated using four far-field beacons. The calibration equation (4) is applied with no prior knowledge on $\Delta \mathbf{P}$ with the exception of the reference antenna. Fig. 9 shows the residual standard deviation of the calibrated parameters normalized to $\sigma_{\Delta \Phi}$, together with the covariances as described above. Again, $\Delta z$ is strongly coupled with $\Delta \phi$, and the variance of the difference is small compared to $\sigma_{\Delta x}$ and $\sigma_{\Delta y}$, which are independent.

To limit the norm of $\sigma_{\Delta x}, \sigma_{\Delta y}$, and $\operatorname{std}(\Delta z-\Delta \phi)$ to $1 \times 10^{-4} \lambda$, the required $\sigma_{\Delta \Phi}$ is $1.6 \mathrm{mrad}$ for the proposed array, taken from Fig. 9 (right). The same set of beacons defined in Table II is used, to allow both full and truncated routines to be conducted for the proposed mission. In this case, doubling the required calibration time achieves the required $\sigma_{\Delta \Phi}$, as summarized in Table III. The simulated performance of this configuration is shown in Fig. 10. Again, the presented
TABLE III

Example Configuration for Full Receiver Position and Phase CALibration Using Four Far-Field BEACONS

\begin{tabular}{lll}
\hline Parameter & Symbol & Value \\
\hline Cumulative Integration Time per Beacon & $\tau_{\text {far }}$ & $1 \mathrm{~s}$ \\
Total Calibration Time & $\tau_{t o t}$ & $8 \mathrm{~s}$ \\
Visibility Phase Noise & $\sigma_{\Delta \Phi}$ & $1.6 \mathrm{mrad}$ \\
& $\sigma_{\Delta x}$ & $71 \mu \lambda$ \\
Total Calibration Precision & $\sigma_{\Delta y}$ & $71 \mu \lambda$ \\
& $\sigma_{\Delta z}$ & $1.3 \mathrm{m \lambda}$ \\
& $\sigma_{\Delta \phi}$ & $8.3 \mathrm{mrad}$ \\
\hline
\end{tabular}

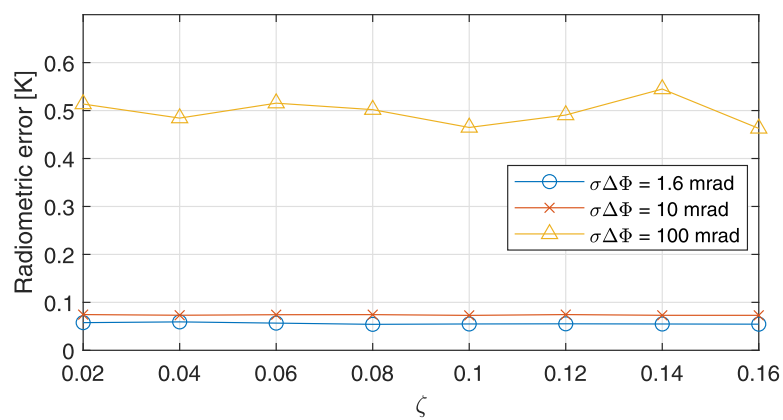

Fig. 10. Simulation results of radiometric accuracy loss caused by various levels of $\sigma_{\Delta \Phi}$ and beacon positions $\zeta$, as shown in Fig. 4, for the full calibration routine (average of 50 Monte Carlo runs). $\sigma_{\Delta \Phi}=1.6 \mathrm{mrad}$ is proposed.

beacon configuration achieves excellent calibration performance, and good performance may still be expected with the beacon requirements relaxed by an order of magnitude.

General comments can now be made on the performance of the presented calibration methods using four airborne beacons on board high altitude balloons. First, note that the coefficient matrix $\mathbf{M}$ (for $\Delta_{\xi}=\Delta \eta=0$ ) is not dependent on antenna positions $(x, y, z)$. This means that the stability achievable in the recovered variables is the same for all antennas, regardless of their positions. Second, the standard deviation of the coupled variable $\Delta z-\Delta \phi$ is constant and is smaller than the $1 \sigma$ of the in-plane antenna positions $\Delta x$ and $\Delta y$ by less than an order of magnitude for the relevant beacon positions $\zeta<$ 0.15 . The effect of out-of-plane errors to the interferometer's radiometric accuracy, however, is stronger than that of in-plane errors by more than an order of magnitude. As a result, the constant $\operatorname{std}(\Delta z-\Delta \phi)$ component has a dominant effect on the radiometric accuracy practically for all $\zeta$. This suggests that there will be little advantage in selecting larger $\zeta$ to minimize the norm shown in Figs. 5 and 9 (right). Doing so will indeed thicken the atmosphere in the beacon signal path length. This is demonstrated in Figs. 7 and 10 .

Finally, the two approaches presented above both fail to achieve good absolute antenna position calibration, as $\Delta z$ and $\Delta \phi$ cannot be decoupled for the proposed geostationary application. The large residual antenna out-of-plane position errors $\Delta z$ is acceptable for microwave interferometry, since these are correlated with the antenna phase errors $\Delta \phi$ to a sufficient degree. On the other hand, for those applications which require precise position calibration, it will be important that the sources are distributed across a diverse set of incidence angles to the interferometer. Section V-C describes the cases where the beacons are distributed on board the 
formation-flying satellites, achieving extremely large $\zeta$ for absolute position calibration.

\section{Absolute Position and Receiver Phase Calibration Using Airborne Beacons and Near-Field Beacons}

The free-flying companion antennas are now allowed to illuminate the multisatellite array to achieve high precision antenna position and phase calibration. Again, this method requires that the phase beam pattern of all antennas at extreme off-axis angles are known to within a few degrees. While further work will be necessary to establish this condition, as discussed further in Section VI, this section discusses the advantages of using near-field beacons to calibrate the absolute positions of the free-flying companion antennas, presented as a concept for future implementation.

The calibration is implemented by allowing each of the nine companion antennas, as well as the central antenna on the central satellite to illuminate the array one at a time, in addition to two far-field beacons on the Earth. As with the previous section, this is applied in two calibration routines: full and truncated.

The number of unknowns in this configuration is $4 N_{1}+N_{2}$, where $N_{1}$ is the number of antennas the position and phase of which are being calibrated, and $N_{2}$ is the number of antennas with known positions and the phase of which is being calibrated. $N_{1}$ and $N_{2}$ are determined by the two different calibration scenarios.

The full calibration takes $N_{1}$ as the total number of antennas less the reference antenna, and $N_{2}=0$. This leaves the coefficient matrix $\mathbf{M}$ underdetermined by three degrees of freedom for orientation, which are constrained by introducing two airborne beacons of known locations.

The truncated calibration takes $N_{1}$ as the number of companion satellites while $N_{2}$ is the number of antennas within the central array, less the reference antenna. The array is illuminated by the nine companion satellites and the reference antenna. The difference to the full calibration routine is that the knowledge of the positions of the central antennas reduces the minimum required number of airborne beacons to just one.

The robustness of the two routines is now discussed.

1) Full Calibration Using Near-Field Beacons: The geometry of the array with ten transmitting beacons is shown in Fig. 1, and the distribution of the two far-field beacons are shown in Fig. 4 (right). With this configuration, the achievable calibration precision again improves as the airborne beacons are separated by larger angles, as shown in Fig. 11.

In this plot, several properties of this calibration technique can be noted. First, $\sigma_{\Delta \phi}$ and $\sigma_{\Delta z}$ are at the same levels as $\sigma_{\Delta x}$ and $\sigma_{\Delta y}$, indicating that the two variables are successfully decoupled with the introduction of near-field beacons. Note especially for the companion antennas near the constellation center (circles) that the achieved $\sigma_{\Delta \phi}$ are much better than $\sigma_{\Delta z}$, showing again the benefit of illuminating the antenna from a diverse set of angles. For all $\zeta$, the achieved robustness is better than that achievable with the previous methods.

Another point to note is that the set of residual errors is unique to each antenna. These residuals are determined by
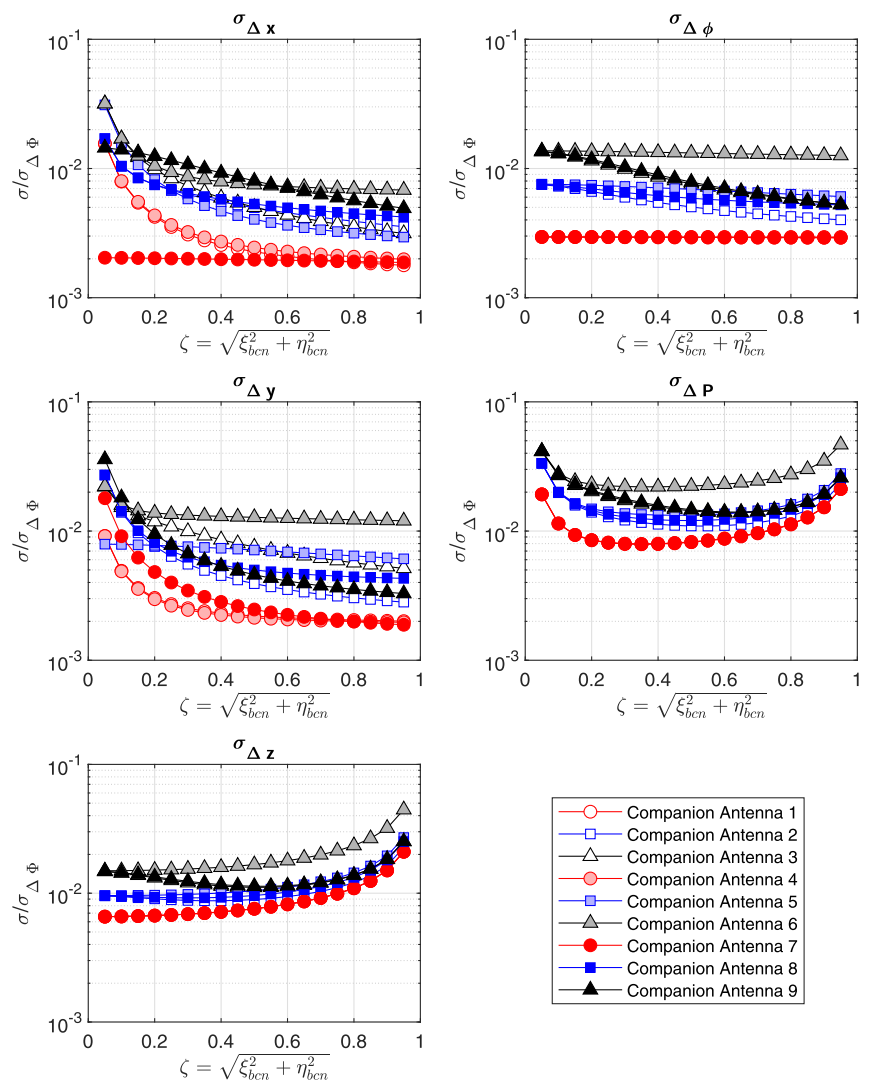

Fig. 11. Achievable calibration precision per measured visibility phase uncertainty for all nine companion satellites. Note $\sigma_{\Delta z}$ and $\sigma_{\Delta \phi}$ are at the same level as the rest, an improvement compared to Figs. 5 and 9.

the distribution of the far-field beacons with respect to the calibrated array, and they are unique because the selected beacon configuration [see Fig. 4 (right)] does not share any common symmetries with the antenna distribution. If a three-beacon triangular configuration is used, for example, then the beacon-antenna system will have $120^{\circ}$ rotational symmetry, and the same calibration precision is achieved within the groups of antennas $(1,4,7),(2,5,8)$, and $(3,6,9)$. Nevertheless, in general, the antennas closer to the center (circles) achieve better calibration precision than those furthest (triangles), as the antennas near the center are illuminated from all directions, while the antennas at the outer edge are illuminated only from one hemisphere.

The effects of residual thermal $\sigma_{\Delta \Phi}$ to the recovered parameters are now discussed. In contrast to the earlier calibration routines, $\sigma_{\Delta \Phi}$ is no longer constant across all baselines, since the power received from the near-field beacons depends on the specific configuration of the beacon and the receiving antenna pair. Taking this into account, in order to achieve $1 \times 10^{-4} \lambda$ precision in the antenna position for the proposed array, the proposed parameters are shown in Table IV.

The propagated uncertainties in the recovered parameters for the companion antennas are then shown in Fig. 12(a). Fig. 12(b) summarizes the norm of $\sigma_{\Delta x}, \sigma_{\Delta y}$, and $\sigma_{\Delta z}$, for each antenna against its distance to the array center.

2) Truncated Calibration Using Near-Field Beacons: The required calibration time for the absolute antenna position for the free-flying companion satellites can be further improved 
TABLE IV

EXAMPle CONFIGURATion For THE Full CALiBRation Routine Using TEn NeAR-Field BEaCONS AND Two Airborne BeACONS

\begin{tabular}{lll}
\hline Parameter & Symbol & Value \\
\hline Far-field Transmitter Diameter & $D_{T_{\text {far }}}$ & $0.5 \mathrm{~m}$ \\
Far-field Transmitter Antenna Gain & $G_{T_{\text {far }}}$ & $48.9 \mathrm{dBi}$ \\
Far-field Transmitter Power & $P_{T_{\text {far }}}$ & $15 \mathrm{~W}$ \\
Far-field Cumulative Integration Time & $\tau_{\text {far }}$ & $5 \mathrm{~s}$ \\
per Beacon & $P_{T_{\text {near }}}$ & $30 \mathrm{~mW}$ \\
Near-field Transmitter Antenna Power & & $1 \mathrm{~s}$ \\
Near-field Cumulative Integration Time & $\tau_{\text {near }}$ & $30 \mathrm{~s}$ \\
per Beacon & $\tau_{\text {tot }}$ & $65 \mu \lambda$ \\
Total Calibration Time & $\sigma_{\Delta x}$ & $71 \mu \lambda$ \\
& $\sigma_{\Delta y}$ & $53 \mu \lambda$ \\
Total Calibration Precision & $\sigma_{\Delta z}$ & $0.24 \mathrm{mrad}$ \\
\hline
\end{tabular}

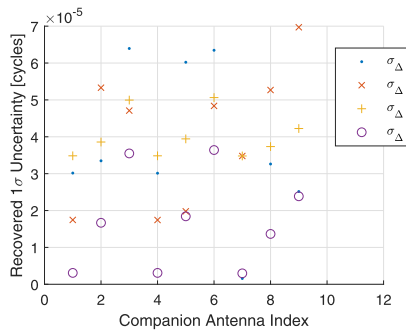

(a)

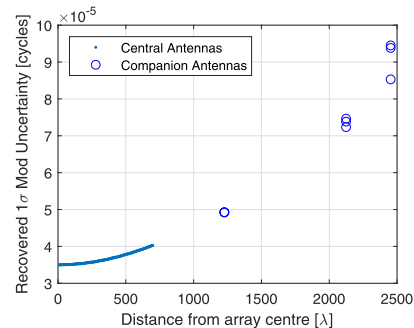

(b)
Fig. 12. Calibration precision achieved with configuration in Table IV. Listed by (a) antenna index and (b) antenna's distance from array center.

TABLE V

EXAMPle CONFIGURATION FOR THE TRUnCATED CALIBRATION Routine With TEN NEAR-FiEld BEACONS AND ONE AIRBorne BEACON

\begin{tabular}{lll}
\hline Parameter & Symbol & Value \\
\hline $\begin{array}{l}\text { Far-field Cumulative Integration Time } \\
\text { per Beacon }\end{array}$ & $\tau_{\text {far }}$ & $5 \mathrm{~s}$ \\
Near-field Cumulative Integration Time & & \\
per Beacon & $\tau_{\text {near }}$ & $0.1 \mathrm{~s}$ \\
Total Calibration Time & $\tau_{\text {tot }}$ & $12 \mathrm{~s}$ \\
& $\sigma_{\Delta x}$ & $53 \mu \lambda$ \\
Total Calibration Precision & $\sigma_{\Delta y}$ & $46 \mu \lambda$ \\
& $\sigma_{\Delta z}$ & $61 \mu \lambda$ \\
& $\sigma_{\Delta \phi}$ & $0.32 \mathrm{mrad}$ \\
\hline
\end{tabular}

if the positions of the antennas within the central array are accurately known. The number of unknowns in this case is $4 N_{1}+N_{2}$, where $N_{1}$ is the number of companion antennas and $N_{2}$ is the number of antennas in the central array, excluding the reference central antenna.

In principle, the coefficient matrix $\mathbf{M}$ in this case can be fully constrained without any far-field beacons, since the exact locations of some of the antennas are provided. On the other hand, since these antennas occupy a plane with very small $z$ components, $\mathbf{M}$ is ill-conditioned unless at least one antenna or beacon is given outside of this plane. For this task, one far-field beacon on the Earth at the center of field of view is selected to fully constrain the coefficient matrix $\mathbf{M}$.

With the antennas distributed as shown in Fig. 1, and with the beacons characterized in Table IV, it is found that integration time of $0.1 \mathrm{~s}$ for the near-field beacons is sufficient to achieve the same level of precision in the recovered companion antenna positions and phase. The calibration parameters for the truncated case are shown in Table $\mathrm{V}$ for the full-sized array,

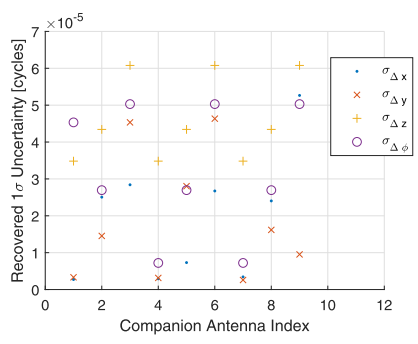

(a)

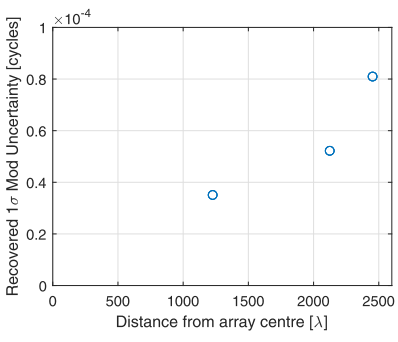

(b)
Fig. 13. Calibration precision achieved with configuration suggested in Table $\mathrm{V}$ for the nine companion satellites. Listed by (a) antenna index and (b) antenna's distance from the array center.

with the achieved calibration precision shown in Fig. 13. Again, antennas closer to the array center achieve a better calibration precision, as shown in Fig. 13(b).

\section{CONCLUSION}

This article has developed calibration techniques suitable for formation-flying interferometric radiometers. The method uses a set of microwave beacons placed at known positions to inject correlated signals into the antennas, where the conventional noise distribution technique using network of cables cannot be applied on free-flying baselines. These signals are separated from the signals generated by the Earth's brightness by taking two successive exposures of the scene: the first with the beacon included, and the second with the beacon switched off. The difference in the visibility measured in these two exposures is the response to the beacon plus the thermal noise associated with finite integration time. This technique allows the calibration of the receiver relative phase error and the antenna position simultaneously.

Calibration equations for two types of beacons have been presented: the far-field beacons which are deployed at known locations at high altitudes in the Earth's atmosphere to avoid severe atmospheric attenuation at the sounding frequencies, and the near-field beacons which are positioned on all of the formation-flying satellites. Using these beacons, five calibration routines have been described.

1) Phase-only calibration using far-field beacons, in which the position of all antennas is known a priori. The phase of all antennas can be calibrated using a single far-field beacon flown in the Earth's atmosphere.

2) Full calibration using far-field beacons, in which the position and phase of all antennas are calibrated using four far-field beacons.

3) Truncated calibration using far-field beacons, in which the antenna positions within the central array are known a priori, and the remaining parameters are calibrated using four far-field beacons.

4) Full calibration using near-field beacons, in which the position and phase of all antennas are calibrated using two far-field beacons and ten near-field beacons, which use some of the receiver antennas to transmit the beacon signals.

5) Truncated calibration using near-field beacons, in which the antenna positions on the central antenna are known 
a priori, and the remaining parameters can be calibrated using ten near-field beacons and a single far-field beacon.

This article has evaluated the performance of these calibration routines applied to a multisatellite geostationary atmospheric sounder concept and found that techniques using far-field beacons ( 2 and 3 ) will not be able to calibrate the antenna out-of-plane position with good accuracy. This is due to the fact that all four beacons occupy a narrow field of view that is the Earth seen from the geostationary orbit, which leaves the interferometer struggling to distinguish between the effects of out-of-plane antenna position error and that of antenna phase error. Nevertheless, the fact that these two errors are strongly coupled means that their combined effects on radiometric accuracy will be minimal. Interferometer simulation runs have verified this, showing that end-to-end radiometric accuracy of better than $0.1 \mathrm{~K}$ is readily achievable. This suggests that this approach will be ideal for geostationary interferometers. Further considerations will be necessary for applications in lower orbits, as a minimum of four beacons must remain within the interferometer's field of view at all times. The proposed parameters for the required far-field beacons are shown in Tables II and III, and the simulated radiometric accuracy are shown in Figs. 7 and 10.

On the other hand, calibration routines using near-field beacons (4 and 5) were found to successfully decouple antenna out-of-plane position with antenna phase, suggesting that this approach will be ideal for high precision absolute position calibration. Major practical challenges remain for implementing this approach, however, namely the design of antennas with phase patterns that are known and stable to within a few degrees at $90^{\circ}$ off-axis angles. An alternative approach will be to employ a separate set of antennas with well-behaving beam patterns at large off-axis angles, e.g., dipole antennas. In this case, the position variability of these antennas with their corresponding main antennas must also be taken into account, or minimized by minimizing the distance between the pair of antennas, and feeding the signals into the same receivers, corresponding to the main antennas.

\section{ACKNOWLEDGMENT}

The authors would like to thank the anonymous reviewers, who helped substantially improve the quality of this article.

All data supporting this article are openly available from the University of Southampton repository at https://doi.org/ 10.5258/SOTON/D1197.

\section{REFERENCES}

[1] F. Ulaby, R. Moore, and A. Fung, Microwave Remote Sensing: Active and Passive, vol. 1. Norwood, MA, USA: Artech House, 1981.

[2] M. J. Mcfarland, R. L. Miller, and C. M. U. Neale, "Land Surface Temperature derived from the SSM/I passive microwave brightness temperatures," IEEE Trans. Geosci. Remote Sens., vol. 28, no. 5, pp. 839-845, Sep. 1990.

[3] T. Kawanishi et al., "The advanced microwave scanning radiometer for the earth observing system (AMSR-E), NASDA's contribution to the EOS for global energy and water cycle studies," IEEE Trans. Geosci. Remote Sens., vol. 41, no. 2, pp. 184-194, Feb. 2003.
[4] S. K. Chan et al., "Assessment of the SMAP passive soil moisture product," IEEE Trans. Geosci. Remote Sens., vol. 54, no. 8, pp. 4994-5007, Aug. 2016.

[5] J. Font et al., "SMOS: The challenging sea surface salinity measurement from space," Proc. IEEE, vol. 98, no. 5, pp. 649-665, May 2010.

[6] P. W. Gaiser et al., "The WindSat spaceborne polarimetric microwave radiometer: Sensor description and early orbit performance," IEEE Trans. Geosci. Remote Sens., vol. 42, no. 11, pp. 2347-2361, Nov. 2004.

[7] R. R. Ferraro et al., "NOAA operational hydrological products derived from the advanced microwave sounding unit," IEEE Trans. Geosci. Remote Sens., vol. 43, no. 5, pp. 1036-1049, May 2005.

[8] A. R. Thompson, J. M. Moran, and G. W. Swenson, Interferometry and Synthesis in Radio Astronomy, 3rd ed. Cham, Switzerland: Springer, 2017.

[9] F. Torres, A. Camps, J. Bara, I. Corbella, and R. Ferrero, "On-board phase and modulus calibration of large aperture synthesis radiometers: Study applied to MIRAS," IEEE Trans. Geosci. Remote Sens., vol. 34, no. 4, pp. 1000-1009, Jul. 1996.

[10] A. K. S. El Maghraby, A. Grubišic, C. Colombo, and A. Tatnall, "A novel interferometric microwave radiometer concept using satellite formation flight for geostationary atmospheric sounding," IEEE Trans. Geosci. Remote Sens., vol. 56, no. 6, pp. 3487-3498, Jun. 2018.

[11] C. S. Ruf, C. T. Swift, A. B. Tanner, and D. M. Le Vine, "Interferometric synthetic aperture microwave radiometry for the remote sensing of the Earth," IEEE Trans. Geosci. Remote Sens., vol. GRS-26, no. 5, pp. 597-611, Sep. 1988.

[12] I. Corbella, N. Duffo, M. Vall-Llossera, A. Camps, and F. Torres, "The visibility function in interferometric aperture synthesis radiometry," IEEE Trans. Geosci. Remote Sens., vol. 42, no. 8, pp. 1677-1682, Aug. 2004.

[13] R. Butora, M. Martín-Neira, and A. Rivada-Antich, "Fringe-washing function calibration in aperture synthesis microwave radiometry," Radio Sci., vol. 38, no. 2, pp. 15-1-15-15, Apr. 2003.

[14] I. Corbella et al., "MIRAS end-to-end calibration: Application to SMOS L1 processor," IEEE Trans. Geosci. Remote Sens., vol. 43, no. 5, pp. 1126-1134, May 2005.

[15] I. Corbella, F. Torres, A. Camps, and J. Bara, "New calibration technique for interferometric radiometers," Proc. SPIE, Sensors, Syst., Next-Gener. Satell. II, vol. 3498, pp. 359-366, Dec. 1998.

[16] I. Corbella, A. Camps, F. Torres, and J. Bara, "Analysis of noiseinjection networks for interferometric-radiometer calibration," IEEE Trans. Microw. Theory Techn., vol. 48, no. 4, pp. 545-552, Apr. 2000.

[17] O. J. Sovers, J. L. Fanselow, and C. S. Jacobs, "Astrometry and geodesy with radio interferometry: Experiments, models, results," Rev. Mod. Phys., vol. 70, pp. 1393-1454, Oct. 1998.

[18] A.-M. D. Novo-Gradac, G. Yang, S. X. Li, and J. M. Davila, "Relative position sensing for a precision formation flying space telescope," in Proc. 9th Int. Workshop Satell. Constellations Formation Flying, Jun. 2017.

[19] A. Camps, "Application of interferometric radiometry to earth observation," Ph.D. dissertation, Dept. Teoria Senyal Comunicacions, Univ. Politecnica de Catalunya, Barcelona, Spain, 1996, Accessed: Jan. 11, 2019. [Online]. Available: https://www.tdx.cat/handle/10803/6885

[20] J. B. Hagen and D. T. Farley, "Digital-correlation techniques in radio science," Radio Sci., vol. 8, nos. 8-9, pp. 775-784, Aug. 1973.

[21] A. Camps, I. Corbella, J. Bará, and F. Torres, "Radiometric sensitivity computation in aperture synthesis interferometric radiometry," IEEE Trans. Geosci. Remote Sens., vol. 36, no. 2, pp. 680-685, Mar. 1998.

[22] A. Lange et al., "The boomerang experiment," Space Sci. Rev., vol. 74, no. 1, pp. 145-150, Oct. 1995, doi: 10.1007/BF00751263.

[23] E. Pascale et al., "The balloon-borne large aperture submillimeter telescope: Blast," Astrophys. J., vol. 681, no. 1, p. 400, 2008. [Online]. Available: http://stacks.iop.org/0004-637X/681/i=1/a=400

[24] A. Bell et al., "Flight control software for the wave-front sensor of sunrise 1m balloon telescope," Proc. SPIE, vol. 7740, Jul. 2010, Art. no. 774003, doi: 10.1117/12.857003.

[25] F. Ulaby, R. Moore, and A. Fung, Microwave Remote Sensing: Active and Passive, vol. 3. Norwood, MA, USA: Artech House, 1981.

[26] A. B. Tanner et al., "Initial results of the geostationary synthetic thinned array radiometer (GeoSTAR) demonstrator instrument," IEEE Trans. Geosci. Remote Sens., vol. 45, no. 7, pp. 1947-1957, Jul. 2007.

[27] R. Butora, M. Martín-Neira, and A.-L. Rivada-Antich, "Fringe-washing function calibration in aperture synthesis microwave radiometry," Radio Sci., vol. 38, no. 2, pp. 15-1-15-15, 2003. [Online]. Available: https://agupubs.onlinelibrary.wiley.com/doi/abs/10.1029/2002RS002695 
[28] A. Camps, F. Torres, J. Bara, I. Corbella, and F. Monzon, "Automatic calibration of channels frequency response in interferometric radiometers," Electron. Lett., vol. 35, no. 2, pp. 115-116, Jan. 1999.

[29] F. Torres, A. B. Tanner, S. T. Brown, and B. H. Lambrigsten, "Analysis of array distortion in a microwave interferometric radiometer: Application to the GeoSTAR project," IEEE Trans. Geosci. Remote Sens. vol. 45, no. 7, pp. 1958-1966, Jul. 2007.

[30] A. Camps, J. Bara, F. Torres, and I. Corbella, "Extension of the clean technique to the microwave imaging of continuous thermal sources by means of aperture synthesis radiometers," Prog. Electromagn. Res., vols. 19-21, pp. 67-83, 1999, doi: 10.2528/PIER97041500.

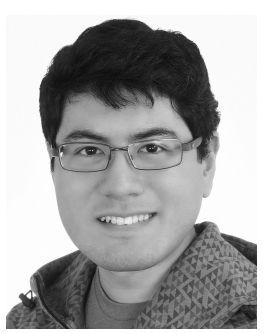

Ahmed Kiyoshi Sugihara El Maghraby received the Master of Engineering degree in space systems engineering from the University of Southampton, Southampton, U.K., in 2014, with a focus on an extremely low-altitude satellite photogrammetry mission concept, where he is currently pursuing the $\mathrm{Ph} . \mathrm{D}$. degree with the Astronautics Research Group.

His research focuses on applying satellite formation flight to passive microwave interferometry, with particular interest in applying this to geostationary microwave radiometry for real-time observation of meteorological phenomena.

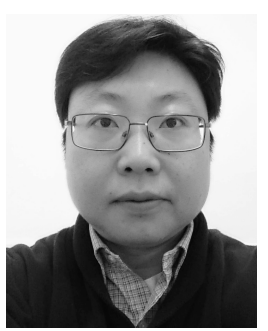

Hyuk Park was born in South Korea. He received the B.S. degree in mechanical engineering from the Korea Advanced Institute of Science and Technology (KAIST), Daejeon, South Korea, in 2001, and the M.S. and Ph.D. degrees in information and mechatronics from the Gwangju Institute of Science and Technology (GIST), Gwangju, South Korea, in 2003 and 2009, respectively.

In 2009, he joined the Remote Sensing Group, Polytechnic University of Catalonia (UPCBarcleonaTech), Barcelona, Spain, as a Post-Doctoral Researcher. He is a grant holder of NRF funded by the Korean Government in 2011. Since 2012, he has been a Juan de la Cierva Fellow funded by the Spanish Ministry of Science and Innovation. He is currently with the CommSensLab, School of Telecommunication and Aerospace Engineering (EETAC), UPC-BarcelonaTech, as a Ramon y Cajal Fellow/Tenure-Track Assistant Professor. His main research interest is in the area of remote sensing and small satellite applications, especially passive microwave remote sensing, including system design, modeling, and simulation, and image processing.

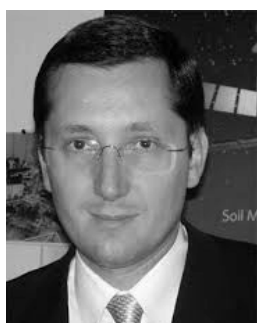

Adriano Camps was born in Barcelona, Spain, in 1969 .

In 1993, he joined the Electromagnetics and Photonics Engineering Group, Department of Signal Theory and Communications, Polytechnic University of Catalonia (UPC), Barcelona, as an Assistant Professor, where he was an Associate Professor in 1997 and has been a Full Professor since 2007. In 1999, he was on sabbatical leave at the Microwave Remote Sensing Laboratory, University of Massachusetts at Amherst, Amherst, MA, USA. He has published over 189 articles in peer-reviewed journals, 6 book chapters, and 1 book (860 pages), and more than 409 international conference presentations, holds 10 patents, and has advised $22 \mathrm{Ph} . \mathrm{D}$. thesis students (+9 on-going), and supervised more than 125 final projects and M.Eng. theses. His research interests are focused on microwave remote sensing, with a special emphasis on microwave radiometry by aperture synthesis techniques (MIRAS instrument onboard ESA's SMOS mission), remote sensing using signals of opportunity (GNSS-R), and nanosatellites as a tool to test innovative remote sensors.

Dr. Camps was the 2017-2018 President of the IEEE Geoscience and Remote Sensing Society.

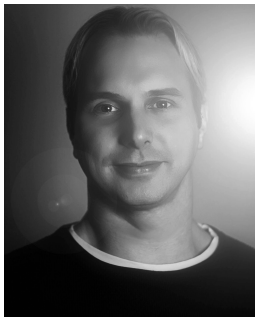

Angelo Grubišić was born in Walsall, U.K., in 1981. He received the M.Sc. degree from the International Space University, Illkirch-Graffenstaden, France, in 2009, and the Ph.D. degree from the University of Southampton, Southampton, U.K., in 2005.

$\mathrm{He}$ has worked extensively on the development and testing of gridded ion thrusters and other electric propulsion systems, including the NSTAR ion thruster at NASA JPL and the T6 ion thruster for QinetiQ. From 2010 to 2013, he was responsible for designing and leading the systems level coupling and qualification tests for the Bepi Colombo electric propulsion system on the Mercury Transfer Module, the highest power dual ion thruster system to date. Since 2013, he has been pioneering selective laser melting additive manufacturing technologies for production of advanced propulsion systems as a Lecturer with the Astronautics Group, University of Southampton. He tragically passed away on the 21 August 2019 in a wingsuit BASE jumping accident.

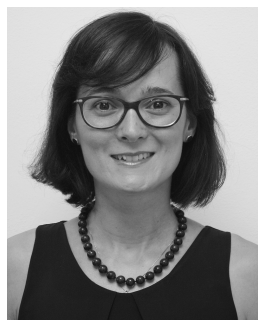

Camilla Colombo received the Ph.D. degree from the University of Glasgow, Glasgow, U.K., in 2010, and the M.Eng. degree in aerospace engineering from the Politecnico di Milano, Milan, Italy, in 2005 .

From 2012 to 2016, she was with the University of Southampton, Southampton, U.K., where she was a Lecturer of spacecraft system engineering courses run at ESA and lead research work on space debris and libration orbit missions. In 2013, she was appointed as a Research Fellow with the Politecnico di Milano, sponsored by a Marie Curie Fellowship. Previously, she was a Research Fellow with the University of Strathclyde, Glasgow, U.K., and a Research Associate with the University of Glasgow, Glasgow, where she was awarded the William Wilson Scott Award Scholarship in 2009 for her Ph.D. thesis on "optimal trajectory design for interception and deflection of near earth objects." She is currently an Associate Professor with the Politecnico di Milano, where she leads the European Research Council Starting Grant on the project "Control for Orbit Manoeuvring through Perturbations for Application to Space Systems" (COMPASS). Her work spans orbital dynamics, space debris, high area-to-mass spacecraft, trajectory design and control, and asteroid deflection and is published in 35 journal articles and 100 conference publications. She is involved in the EC funded project ReDSHIFT and contracts funded by the ESA.

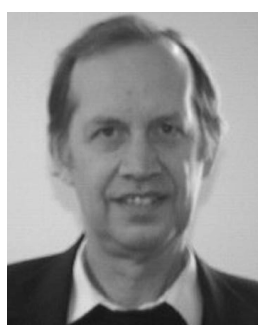

Adrian Tatnall graduated from Exeter University, Exeter, U.K., in 1973, and received the Ph.D. degree from The University of Sheffield, Sheffield, U.K., in 1978 .

He was a Post-Doctoral Research Assistant with York University, York, U.K., and then a Senior Space Systems Engineer with British Aerospace, Farnborough, U.K. He joined the Department of Aeronautics and Astronautics, University of Southampton, Southampton, U.K., in 1988, where he became the Head of the Astronautics Group and a Senior Tutor. His research has included internal waves, ocean color, SAR, formation flying, and neural nets. In addition, he has been directly involved in the design and modeling of the performance of satellite passive microwave instruments. $\mathrm{He}$ was the Organizer and Lecturer of spacecraft system engineering courses run for industry at the European Space Agency and Southampton for 25 years. He has also authored chapters on systems engineering and telecommunications in the book titled Space Systems Engineering edited by Fortescue, Stark, and Swinerd.

Dr. Tatnall served on the Space Committee of the U.K. Royal Aeronautical Society, for many years. 